\title{
Downregulation of PD-L1 via amide analogues of brefelamide: Alternatives to antibody-based cancer immunotherapy
}

\author{
JING ZHANG $^{1}$, OSAMU YAMADA ${ }^{1}$, SHINYA KIDA ${ }^{1}$, SHINYA MURASE ${ }^{1}$, \\ TOSHIO HATTORI ${ }^{2}$, YOSHITERU OSHIMA ${ }^{3}$ and HARUHISA KIKUCHI ${ }^{3}$ \\ ${ }^{1}$ Research and Development Center, FUSO Pharmaceutical Industries, Ltd., Osaka 536-8523; \\ ${ }^{2}$ Department of Health Science and Social Welfare, Kibi International University, Takahashi, Okayama 716-8508; \\ ${ }^{3}$ Laboratory of Natural Products Chemistry, Graduate School of Pharmaceutical Sciences, \\ Tohoku University, Sendai, Miyagi 980-8578, Japan
}

Received July 23, 2019; Accepted January 7, 2020

DOI: $10.3892 / \mathrm{etm} .2020 .8553$

\begin{abstract}
The therapeutic blockade of immune checkpoint has emerged as an effective treatment option for a broad range of tumors. However, the objective tumor response is still limited to a small number of cases and tumor types. The full utility of monoclonal antibody (mAb)-based treatment is hindered by several inherent limitations. Thus, there is an urgent requirement to explore alternative modalities targeting the same pathways. In the present study, two amide analogues of brefelamide, TPFS-201 and TPFS-202, were identified as small molecular immune checkpoint inhibitors, as they downregulated PD-L1 expression in tumor cells. PD-L1 was suppressed in cancer cells treated with TPFD compounds at both mRNA and protein levels, as detected by reverse transcription quantitative PCR and flow cytometric analysis, respectively. Reporter assays using a PD-L1 promoter luciferase construct confirmed the transcriptional inhibition of PD-L1 by TPFS compunds. TPFS compound-mediated PD-L1 downregulation in cancer cells consequently restored $\mathrm{T}$ cell activity, as identified by the reduction of apoptosis and an increase in interleukin-2 promoter activity in Jurkat $\mathrm{T}$ cells, which were co-cultured with TPFS compound-treated A549 cells. TPFS compound-mediated PD-L1 inhibition was partially abolished by the disruption of the putative transcriptional co-activator with PDZ (TAZ)/TEA domain (TEAD)-binding motif in the PD-L1 promoter. The inhibitory effect of TPFS compounds on PD-L1 was markedly inhibited in mouse cell lines, which is consistent with previous research demonstrating that PD-L1 regulation by TAZ is not conserved in mice due to distinct promoter sequences flanking the TAZ/TEAD-binding motif.
\end{abstract}

Correspondence to: Dr Jing Zhang, Research and Development Center, FUSO Pharmaceutical Industries, Ltd., 2-3-30 Morinomiya, Joto-ku, Osaka 536-8523, Japan

E-mail: j-zhang@fuso-pharm.co.jp

Key words: brefelamide, amide analogue, immune checkpoint, programmed death ligand-1, Hippo, TAZ
Together, the data of the current study indicated the potential utility of the brefelamide amide analogues as small molecule immune checkpoint inhibitors, thereby providing therapeutic alternatives, which could be used as monotherapy or in combination with mAbs-based treatment.

\section{Introduction}

As a result of persistent antigen exposure, which occurs in chronic viral infection and cancer, T cells progressively lost their effector functions, entering a state called 'exhaustion' (1-3). Exhausted T cells are characterized by the overexpression of multiple inhibitory receptors (immunological checkpoints), such as programmed death-1 (PD-1, also known as CD279) (4-6). After binding its ligand PD-L1, PD-1 attenuates antigen-specific $\mathrm{T}$ cell response, suppressing the tumor-killing activity of $\mathrm{T}$ cells $(7,8)$. Functional restoration of exhausted T cells by antibodies masking PD-1 on immune effector cells or PD-L1 on tumor cells has produced promising results and has become a significant breakthrough in cancer immunotherapy (9-11). Therapeutic benefits from PD-1/PD-L1 axis blockade have been achieved in patients with an expanding scope of malignancies (12-15). Antibody-based immune checkpoint blockade therapy, however, is expensive to manufacture and administer, and also has several disadvantages such as the lack of oral bioavailability, and a long half-life, which causes difficulties when attempting to remove them from the body, particularly in cases of serious adverse events $(16,17)$. Novel modalities utilizing small molecules targeting the same pathways are thus highly anticipated. The development of small molecules toward these targets, however, is hampered by the limited structural information on these immune checkpoint proteins (18).

PD-L1 is an immune co-regulatory molecule belonging to the B7 family (19). While PD-1 is predominantly expressed on activated T cells, PD-L1 is expressed on the surface of both cancer and immune cells. By triggering an inhibitory signal towards the $\mathrm{T}$ cell receptor-mediated activation, $\mathrm{PD}-\mathrm{L} 1$ suppresses the proliferation, activation and infiltration of cytotoxic T-lymphocytes, consequently facilitating tumor immune escape and cancer progression (20-22). Furthermore, PD-L1 has been reported to be aberrantly overexpressed in 
numerous types of tumor cell, including melanoma, ovarian and lung cancers, and patients with high PD-L1 expression are associated with unfavorable prognosis and significant risk of cancer-specific mortality (23-25). Therefore, pharmaceutical inhibition of PD-L1 expression represents an alternative approach to currently available cancer immunotherapy.

Brefelamide is an aromatic amide isolated from Dictyostelium cellular slim molds. A previous study revealed that brefelamide inhibits the proliferation of human-derived $1321 \mathrm{~N} 1$ astrocytoma cells in response to growth factors (26), and the anti-proliferative effect was associated with a reduction of phosphorylation of extracellular signal-regulated kinase (ERK), AKT and c-jun-N-terminal kinase (27). More recently, our previously published study demonstrated that brefelamide inhibited osteopontin (OPN) expression and OPN-mediated cell invasion of human lung adenocarcinoma-derived A549 cells (28). In the present study amide analogues of brefelamide were found to suppress PD-L1 expression in different cancer cell lines and mitigated PD-1/PD-L1-mediated exhaustion of Jurkat T-lymphocytes co-cultured with A549 cells, and the Hippo pathway is possibly involved in the inhibition of PD-L1 by the amide analogues, which is mediated by a putative binding site for transcriptional co-activator with PDZ (TAZ)/Yes-associated protein (YAP)-TEA domain (TEAD) on PD-L1 promoter.

\section{Materials and methods}

Plasmids. For construction of the reporter vector pPD-L1-luc, the promoter sequence from -2094 to +54 was amplified by PCR using human genomic DNA as a template with the following primers: Forward, 5'-ataggtaccACTGCTCTT CTCCCATCTCA-3' and reverse 5'-ataccatggtggctttaccaa cagtaccggattgccaagcttAAGCTGCGCAGAACTGGGGC-3'. The amplified products were digested with Kpn $I$ and Nco I, and subsequently ligated with pGL3-basic (Promega Corporation) which has previously digested with the same enzymes. pmTREPD-L1-luc and pdTREPD-L1-luc are identical to pPD-L1-luc, except for the mutation or deletion introduced to disrupt the TEAD responsive element (TRE), respectively, which were generated using PCR site-directed mutagenesis. Briefly, using pPD-L1-luc as the template, the proximal part of the PD-L1 promoter sequence was amplified using 5 ' primer containing either the mutation (5'-TGAAAG CTTCCGCCGATTTCACCGAAGGTCTCCTTTCTCCAA CGCCCGGCAAACTGGATTTG-3' for pmTREPD-L1-luc) or deletion (5'-TGAAAGCTTCCGCCGATT TCACC G A AG GTC GCC CGG CAA ACT GGA T TTG-3' for pdTREPD-L1-luc) and the aforementioned reverse primer. The resultant products were then digested with HindIII and NcoI and inserted into pPD-L1-luc, which was previously digested with the same enzymes. The sequence of the PCR-manipulated regions and the presence of the expected mutations or deletions were confirmed by nucleotide sequencing.

Cells. The human lung adenocarcinoma-derived A549 cells and the PC-3 human prostate cancer cell line were purchased from American Type Culture Collection and maintained in Dulbecco's modified Eagle's medium (Invitrogen: Thermo Fisher Scientific, Inc.) supplemented with $10 \%$ fetal bovine serum (FBS) and $50 \mathrm{U} / \mathrm{ml}$ penicillin and streptomycin at $37^{\circ} \mathrm{C}$ in a humidified incubator with $5 \% \mathrm{CO}_{2}$. The THP-1 human leukemia monocytic cell line and the Jurkat T-lymphocytes were maintained in RPMI1640 supplemented with $10 \%$ FBS. The cell line, A549/PD-L1-luc, was established by co-transfection of A549 cells with pPD-L1-luc and pPUR (Clontech Laboratories, Inc.), followed by selection in the presence of $1 \mu \mathrm{g} / \mathrm{ml}$ puromycin (Sigma-Aldrich; Merck KGaA).

Cytokines, reagents and antibodies. Recombinant human interferon (IFN) $\gamma$ was purchased from PeproTech, phorbol 12-myristate 13-acetate (PMA), and phytohemagglutinin (PHA) were purchased from Sigma-Aldrich (Merck KGaA), and Roche Diagnostics, respectively. Anti-human PD-1 antibody and recombinant PD-L1 were purchased from R\&D Systems, Inc.

Chemicals. Amide analogues of brefelamide were synthesized by maintaining the position of the benzene rings, the carbonyl groups and the amino groups contained in brefelamide for the purpose of focusing on the suppressive effect of brefelamide on PD-L1 expression. TPFS-201 and TPFS-202 (Fig. 1A) are the representative amide analogues. They were dissolved in DMSO at a concentration of $50 \mathrm{mmol} / 1$ and store at $-20^{\circ} \mathrm{C}$. Aliquots of the stock solution were subsequently diluted to the indicated concentration before treatment of the cells.

Reverse transcription-quantitative PCR (RT-qPCR). Cells were lysed with TRIzol ${ }^{\circledR}$ reagent (Invitrogen; Thermo Fisher Scientific, Inc.), and total RNA was extracted according to the manufacturer's instructions. After treatment with RNase-free DNase (Promega Corporation), the DNA-free RNA (250 ng) was used for synthesis of the first-strand cDNA at $42^{\circ} \mathrm{C}$ for 60 min using MMLV reverse transcriptase (Invitrogen; Thermo Fisher Scientific, Inc.). RT-qPCR was performed using Power SYBR Green PCR Master Mix (Applied Biosystems; Thermo Fisher Scientific, Inc.) at $95^{\circ} \mathrm{C}$ for $15 \mathrm{~s}$ and at $60^{\circ} \mathrm{C}$ for $1 \mathrm{~min}$, for 40 cycles, in a 96-well format on StepOnePlus ${ }^{\mathrm{TM}}$ Real-Time PCR Systems (Applied Biosystems; Thermo Fisher Scientific, Inc.). The following primer sequences were used: PD-L1 forward, 5'-GGCATTTGCTGAACGCAT-3', and reverse, 5'-CAATTAGTGCAGCCAGGT-3'; GAPDH forward, 5'-TGATGACATCAAGAAGGTGG-3', and reverse, 5'-TCC TTGGAGGCCATGTGGGC-3'.

Flow cytometric analysis. Surface expression of PD-L1 was assessed by flow cytometry. Briefly, cell suspensions were prepared and washed in fluorescence-activated cell sorter buffer consisting of phosphate-buffered saline containing $1 \%$ bovine serum albumin. After blocking with normal mouse serum, cells were incubated with fluorochrome (PC7) labeled anti-CD274 antibody (cat. no. A78884; Beckman Coulter, Inc.) or mouse IgG1 isotype control (cat. no. 737662; Beckman Coulter, Inc.) in the dark for $30 \mathrm{~min}$, in an ice bath. After fixation, the fluorescent signals from cells were acquired on a Cell Sorter SH800 flow cytometer (Sony Europe B.V.) and the data was analyzed using FlowJo software (BD Biosciences). To determine the surface expression of PD-L1 in mouse cells, PE labeled anti-mouse CD274 antibody (cat. no. 12-5982-82) or rat IgG2a $\kappa$ (cat. no. 12-4321-80; both Thermo Fisher Scientific, Inc.) was used. 
A

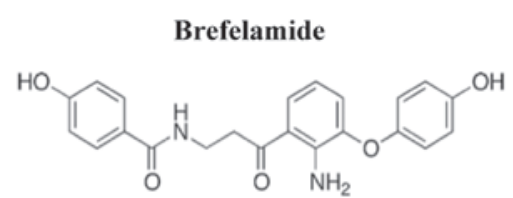

TPFS-201

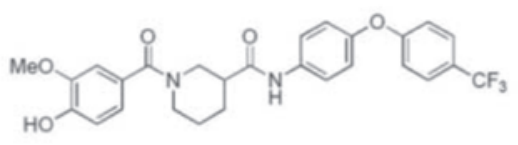

TPFS-202

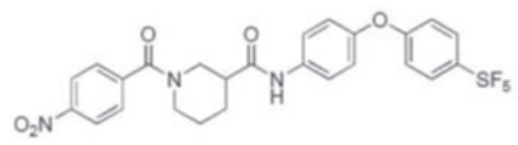

B

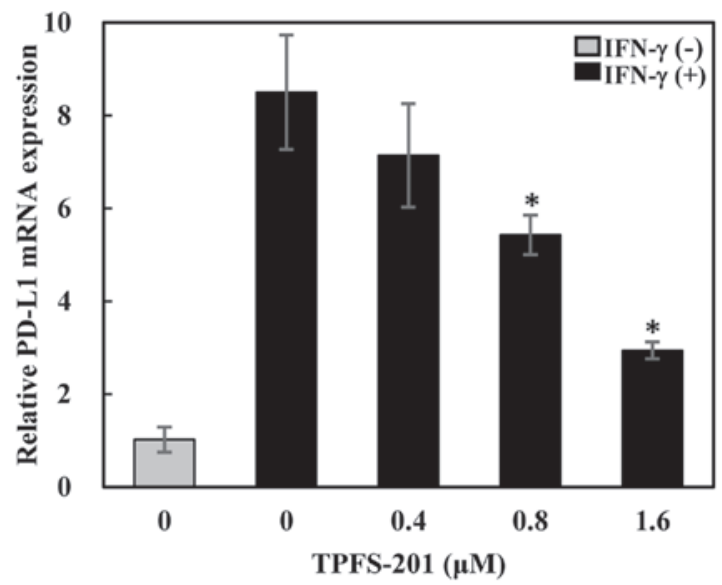

$\mathrm{C}$

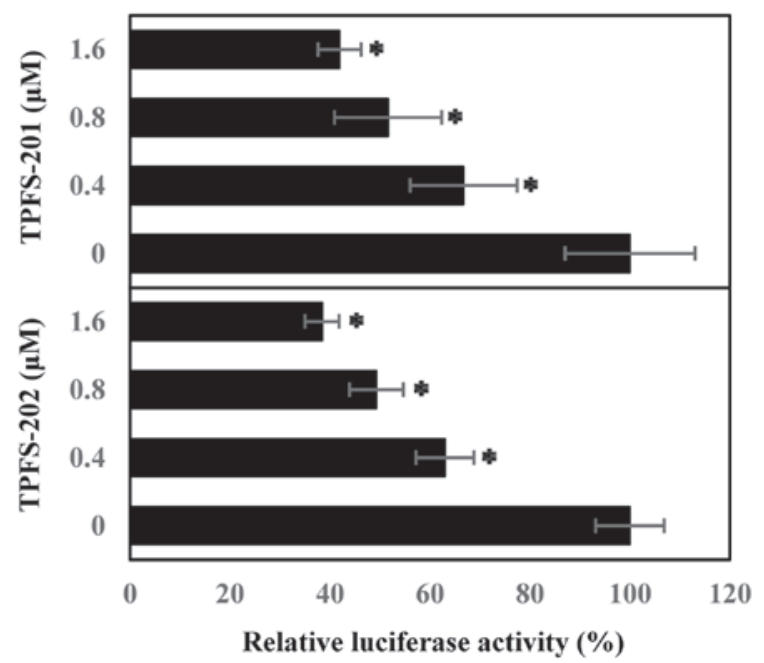

Figure 1. Suppression of PD-L1 transcription by TPFS compounds. (A) Structures of brefelamide and its derivative TPFS compounds. (B) PC-3 cells were stimulated with IFN- $\gamma$ and cultured in the absence or presence of various concentrations of TPFS-201 for $24 \mathrm{~h}$. RNA was subsequently prepared and subjected to reverse transcription-quantitative PCR. All samples were normalized to the expression of GAPDH. (C) A549 cells, stably transfected with pPD-L1-luc, were either untreated or treated with different concentrations of TPFS-201 or TPFS-202. After $48 \mathrm{~h}$ of culture, cells were harvested and lysates were subjected to the luciferase assay and protein determination. The luciferase values were normalized with respect to protein content. Normalized luciferase activity from mock-treated cells was set as $100 \%$ and values in the experimental groups were expressed as relative percentage. Results are presented as the mean \pm standard deviation of three independent triplicate measurements. ${ }^{*} \mathrm{P}<0.05$ vs. vehicle-treated controls. PD-L1, programmed death-ligand 1; IFN, interferon.
Luciferase assay. A549/PD-L1-luc cells were cultured for $48 \mathrm{~h}$ at various concentrations of TPFS compounds. The cells were harvested, and the cell lysates were prepared for the luciferase assay with the Luciferase Reporter Assay System (Promega Corporation) according to the manufacturer's protocol. Luciferase activities were measured using a GloMax ${ }^{\circledR} 20 / 20$ Luminometer (Promega Corporation). The protein concentration in the cell lysate was determined with Pierce BCA Protein Assay kit (Thermo Fisher Scientific, Inc.) according to the manufacture's protocol. The luciferase values were normalized to protein content.

T cell apoptosis assays. T cell apoptosis assays were performed as described previously (29). Briefly, A549 cells were treated with interferon (IFN)- $\gamma$ alone or together with TPFS-201 for $48 \mathrm{~h}$. Jurkat T cells were activated with $0.25 \mu \mathrm{g} / \mathrm{ml}$ PHA and $12.5 \mathrm{ng} / \mathrm{ml}$ PMA for $48 \mathrm{~h}$ to induce PD-1 expression. IFN- $\gamma$ and/or TPFS-201-treated A549 cells were co-cultured with PHA- PMA-activated Jurkat cells at a ratio of 10:1. After $24 \mathrm{~h}$, apoptosis in Jurkat cells was measured using the Caspase-Glo ${ }^{\circledR}$ 3/7 Assay Systems (Promega Corporation). In parallel experiments, Jurkat T cells were pre-incubated with $10 \mu \mathrm{g} / \mathrm{ml}$ anti-PD-1 antibody (R\&D Systems) or mouse IgG isotype control for $3 \mathrm{~h}$ prior to co-culturing with IFN- $\gamma$-stimulated A549 cells.

$T$ cell activation bioassay. Jurkat $\mathrm{T}$ cells were transfected with an interleukin (IL)-2 reporter plasmid, pIL2-luc, using TransIT-Jurkat Transfection Reagent (Mirus Bio LLC). The cells were stimulated with $0.25 \mu \mathrm{g} / \mathrm{ml}$ PHA and $12.5 \mathrm{ng} / \mathrm{ml}$ PMA to initiate PD-1 expression, $24 \mathrm{~h}$ after transfection. A549 cells were treated with IFN- $\gamma$ alone or together with TPFS-201 for $48 \mathrm{~h}$. IFN- $\gamma$ - and/or TPFS-201-treated A549 cells were co-cultured with PHA- and PMA-activated Jurkat T cells at an effector-to-target ratio of 10:1. In a parallel experiment, PHA- and PMA-activated Jurkat T cells were preincubated with $10 \mu \mathrm{g} / \mathrm{ml}$ anti-PD-1 for $3 \mathrm{~h}$ before co-culturing with IFN- $\gamma$-stimulated A549 cells. In a further experiment, IFN- $\gamma$ - and TPFS-treated A549 cells were co-cultured with Jurkat T cells in the presence of $10 \mu \mathrm{g} / \mathrm{ml}$ recombinant human PD-L1/B7-H1 Fc (R\&D Systems). Jurkat T cells were collected $24 \mathrm{~h}$ later, and activities of luciferase driven by IL-2 promoter were measured.

Statistical analysis. Experimental data are presented as the mean \pm standard deviation from three or more independent experiments. All P-values were determined using one-way ANOVA followed by Dunnett's multiple comparisons test. Data were analyzed using Statcel 4 software (OMS, Inc.) $\mathrm{P}<0.05$ was considered to indicate a statistically significant difference.

\section{Results}

Inhibition of PD-L1 expression by TPFS-201 and TPFS-202. While the regulation of PD-L1 expression is yet to be elucidated, transcription of PD-L1 has been reported to be activated in response to differentsignaling pathways including EGF-induced PI3K/AKT and RTK/Ras/MAPK signaling (30-32). Considering that multiple target genes downstream of these pathways were downregulated in microarray-based gene 

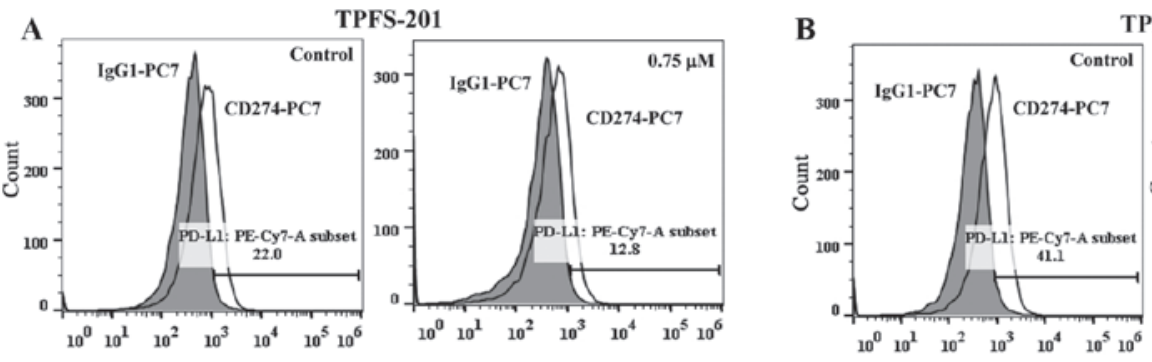

TPFS-202
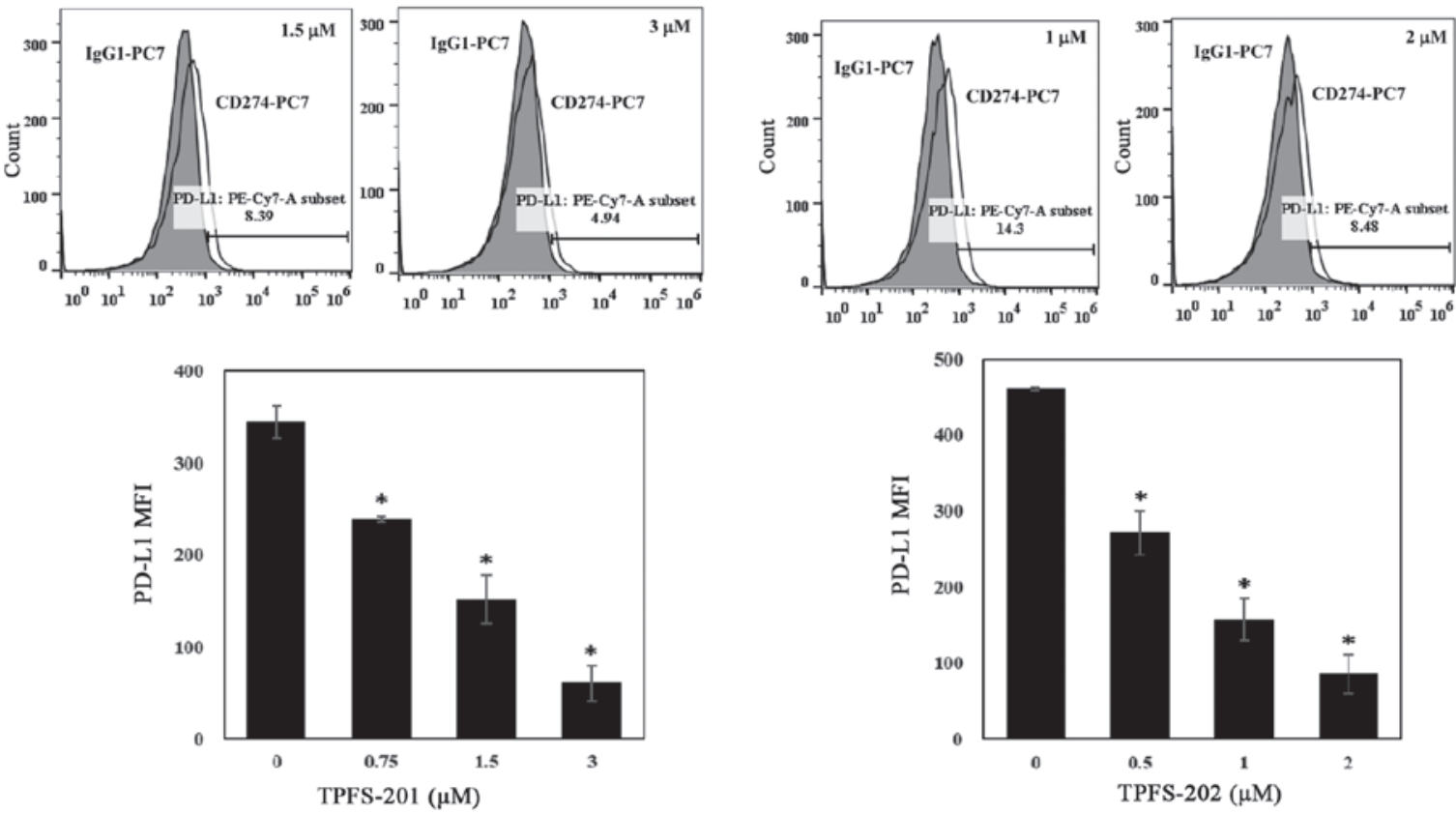

Figure 2. Inhibition of cell surface PD-L1 protein expression via TPFS compounds. Representative flow cytometry histograms (upper) and MFIs (lower) of PD-L1 on THP-1 cells untreated or treated with the indicated concentrations of (A) TPFS-201 or (B) TPSF-202 (n=3 independent experiments). The THP-1 human monocytic leukemia cell line was treated with the indicated concentrations of TPFS compounds for $48 \mathrm{~h}$ in the presence of interferon- $\gamma$. Following treatment, cells were harvested and PD-L1 was analyzed using flow cytometry. All MFI values were averaged from three independent measurements. " $\mathrm{P}<0.05$ vs. untreated controls. MFI, median fluorescence intensity; PD-L1, programmed death-ligand 1.

profiling analysis of brefelamide-treated A549 cells (28), it was hypothesized whether brefelamide or its amide analogues could suppress PD-L1 expression in tumor cells, thereby promoting $\mathrm{T}$ cell-mediated antitumor immunity. To address this issue, the effect of brefelamide amide analogues on PD-L1 mRNA expression was investigated. Following culturing in the presence of IFN- $\gamma$ alone or in combination with TPFS-201, RNA from PC-3 was extracted and the PD-L1 mRNA level was determined using RT-qPCR. As shown in Fig. 1B, IFN- $\gamma$ stimulation markedly increased PD-L1 mRNA level in PC-3 cells, and interestingly, treatment with TPFS-201 dose-dependently reduced IFN- $\gamma$-induced PD-L1 mRNA expression, while basal PD-L1 expression levels were not affected. To further characterize the effect of TPFS-201 on PD-L1 transcription, an experimental system was created, using the A549 cell line stably transfected with pPD-L1-luc, in which the luciferase gene is under the control of human PD-L1 promoter. After treatment for $48 \mathrm{~h}$, with increasing concentration of TPFS-201 or TPFS-202, luciferase expression in A549/PD-L1-luc cells was suppressed in a concentration-dependent manner (Fig. 1C), confirming a transcriptional repression of PD-L1 by the amide analogues of brefelamide. The consistent change in PD-L1 expression was also identified in the flow cytometric analysis, in which there was a 31, 56 and $83 \%$ decrease in the median fluorescence intensity of THP-1 cells treated with TPFS-201 at the concentration of $0.75,1.5$ and $3 \mu \mathrm{M}$, respectively (Fig. 2A), and a 41, 66 and $81 \%$ decrease in THP-1 cells treated with TPFS-202 at the concentration of $0.5,1$ and $2 \mu \mathrm{M}$, respectively (Fig. 2B). The $\mathrm{IC}_{50}$ values calculated from dose-response curves were 0.93 and $0.67 \mu \mathrm{M}$ for TPFS-201 and TPFS-202, respectively (Table SI). These results suggest that inhibition of PD-L1 by amide analogues of brefelamide is not cell type specific.

Effect of TPFS compounds on PD-1/PD-L1-mediated immunosuppression. By engaging PD-1 on T cells, tumor-expressed PD-L1 inactivates the anti-tumor response by suppressing T cell proliferation, promoting $\mathrm{T}$ cell apoptosis and inhibiting cytokine production. The therapeutic potential of TPFS-201 and TPFS-202 in restoring anti-cancer immune response by targeting PD-L1 expression was subsequently explored. Co-culture experiment of A549 cells and Jurkat T cells was conducted to investigate if TPFS compound-mediated PD-L1 inhibition could alleviate the function loss of co-cultured Jurkat T cells. Apoptosis and IL-2-directed reporter gene expression (as an indicator of IL-2 production) in co-cultured Jurkat $\mathrm{T}$ cells were measured to monitor $\mathrm{T}$ cell function. Prior to co-culture, A549 cells were stimulated with IFN- $\gamma$ 
A
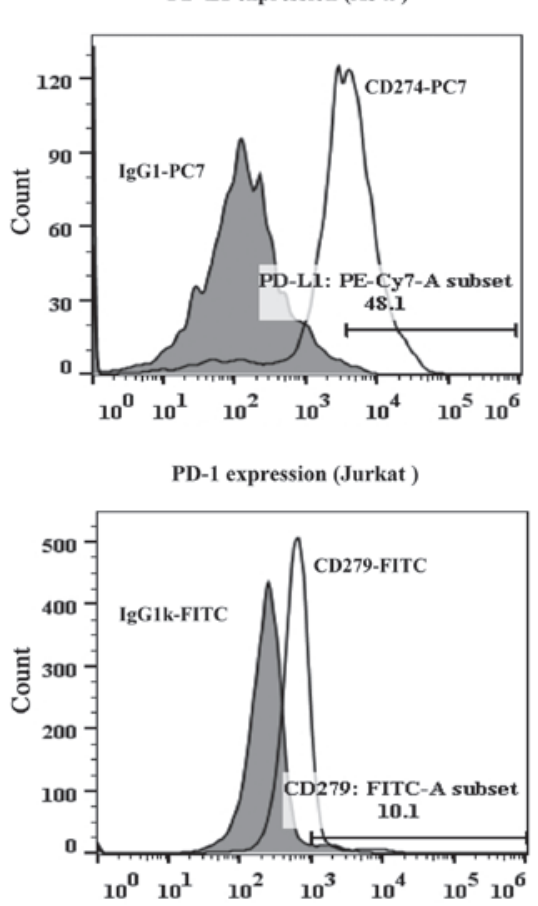

B

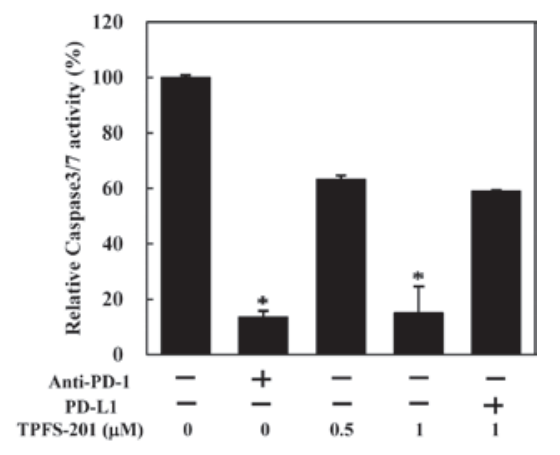

C

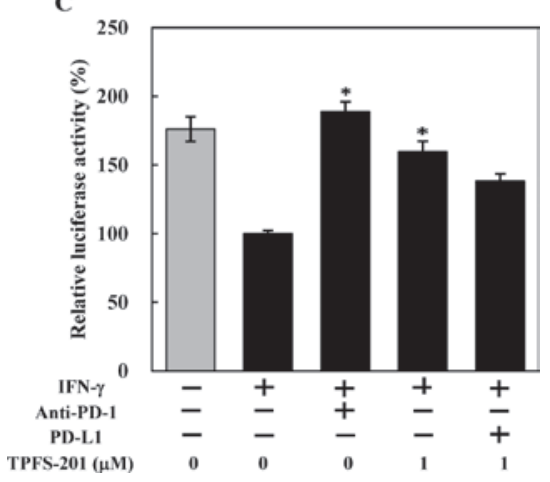

Figure 3. Restoration of T cell function by TPFS-201. (A) PD-L1 expression in A549 cells treated with $100 \mathrm{U} / \mathrm{ml}$ interferon- $\gamma$ (upper). PD-1 expression in Jurkat T cells stimulated with $12.5 \mathrm{ng} / \mathrm{ml}$ PMA and $0.25 \mu \mathrm{g} / \mathrm{ml}$ PHA (lower). (B) Decreased apoptosis and (C) enhanced IL-2-luciferase expression in Jurkat T cells co-cultured with TPFS-201-treated A549 cells. A549 cells were pretreated with interferon- $\gamma$ alone or in combination with TPFS-201 and co-cultured with Jurkat T cells stimulated with $12.5 \mathrm{ng} / \mathrm{ml}$ PHA and $0.25 \mu \mathrm{g} / \mathrm{ml}$ PMA. Caspase 3/7 activities and IL-2-directed luciferase expression were determined as indicators of $\mathrm{T}$ cell function. Caspase 3/7 activities or luciferase expression in Jurkat T cells co-cultured with TPFS-201-treated A549 cells were normalized to that of T cells co-cultured with vehicle-treated A549 cells, which was arbitrarily set as $100 \%$. Results are presented as the mean \pm standard deviation from three independent experiments. ${ }^{*} \mathrm{P}<0.05$ vs. co-cultured vehicle-treated A549 cells. PD-L1, programmed death-ligand 1; PMA, phorbol 12-myristate 13-acetate; PHA, phytohemagglutinin; IL, interleukin. to induce PD-L1 expression, and Jurkat cells were activated with PMA and PHA to induce PD-1 expression (Fig. 3A). As shown in Fig. 3B, A549 cells induced apoptosis in co-cultured Jurkat $\mathrm{T}$ cells, and pre-incubation of Jurkat $\mathrm{T}$ cells with anti-PD-1 antibody disrupting PD-1/PD-L1 interaction prior to co-culture, almost fully abrogated apoptosis in Jurkat T-cells, indicating apoptosis was mediated by PD-1/PD-L1 interaction. Notably, decreases in apoptosis was also observed in Jurkat T cells co-cultured with A549 cells pretreated with TPFS-201. Furthermore, apoptosis inhibition in co-cultured Jurkat T cells by TPFS-201 was negated to some extent by the addition of recombinant human PD-L1 protein, suggesting that TPFS-201 inhibited effector (Jurkat T) cells apoptosis, at least partially, through downregulation of PD-L1 expression on co-cultured target (A549) cells. In addition, co-cultivation with IFN- $\gamma$-stimulated A549 cells inhibited IL-2-luc expression in Jurkat reporter cells, which was restored by pre-incubating with a PD-1 blocking antibody (Fig. 3C). Treatment of A549 cells with TPFS-201 also restored IL-2-luc expression in Jurkat $\mathrm{T}$ cells co-cultured with these cells, albeit less efficiently. Moreover, TPFS-201-mediated restoration of IL-2-luc expression was less evident when co-cultured in the presence of recombinant human PD-L1 protein. Together, these results indicate that TPFS-201-mediated PD-L1 downregulation in cancer cells could partially reverse the functional loss of co-cultured Jurkat $\mathrm{T}$ cells, suggesting a therapeutic potential of the TPFS compound for cancer immunotherapy.

Species-specific inhibition of PD-L1 by TPFS-202. Following the characterized of the inhibitory effect of the brefelamide amide analogues on PD-L1 in vitro, an in vivo evaluation of the efficacy of these compounds as novel immunotherapeutics was subsequently considered. One concern in utilizing a mouse model for drug development, as a prediction of human immunology, is the divergence in the transcriptional programs between the immune systems of the two species, despite a significant similarity in the expression of immune-related genes between the two species (33-35). To explore the feasibility of using immunocompetent syngeneic mouse model for this purpose, it was investigated whether TPFS-202 could also suppresses PD-L1 expression in mouse cancer cell lines. As expected, the B16F10 mouse melanoma cell line efficiently expressed PD-L1 in response to mouse IFN- $\gamma$ stimulation. However, treatment with TPFS-202 only slightly inhibited PD-L1 expression on B16F10 cells (Fig. 4A), with an $\mathrm{IC}_{50}$ value of $6.2 \mu \mathrm{M}$ for TPFS-202, which is 9-fold higher compared with the corresponding value for THP-1 cells. Consistently, RT-qPCR results revealed that treatment with TPFS-201 or TPFS-202 only marginally inhibited PD-L1 mRNA expression in B16F10 and RAW264.7 cells, a murine macrophage cell line (Fig. 4B). The $\mathrm{IC}_{50}$ values of TPFS-201 and TPFS-202 in B16F10 and RAW264.7 cells were 6.4 and $6.1 \mu \mathrm{M}$ and 6.6 and $6.2 \mu \mathrm{M}$, respectively (Table SI). Accordingly, the results show that inhibition of PD-L1 by TPFS compounds is species-specific.

Involvement of Hippo signaling in TPFS-202-mediated PD-L1 suppression. While hypothesizing the molecular basis underlying the observed divergence between human and mouse cells, a study by Janse van Rensburg et al (29) identified PD-L1 as 
A
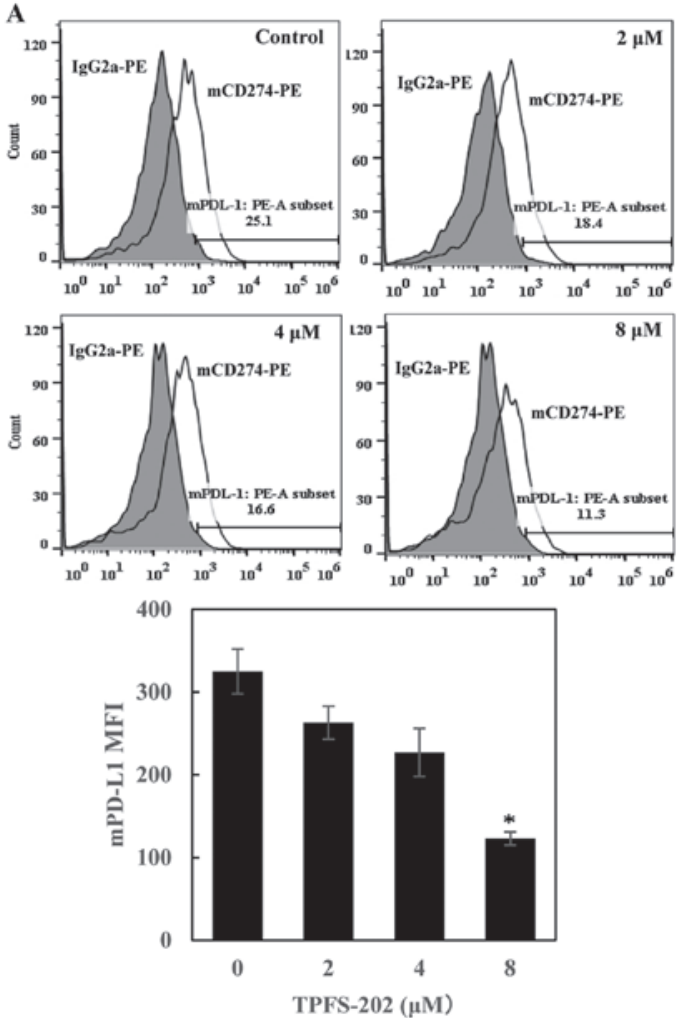

B

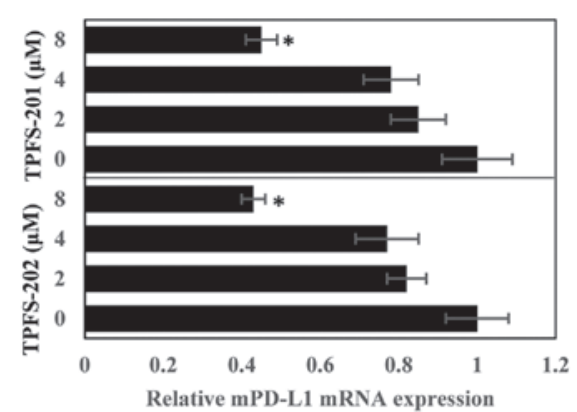

RAW264.7

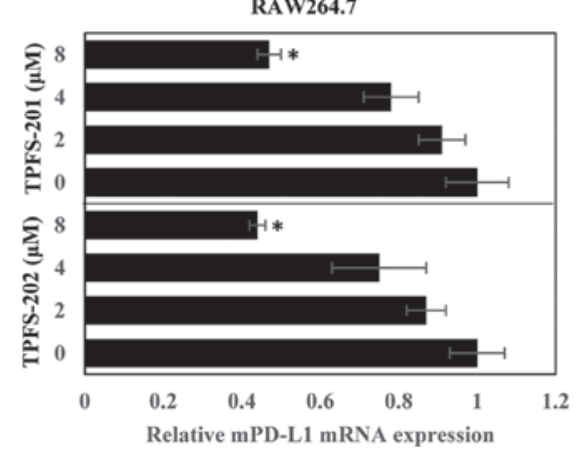

Figure 4. Marginal inhibition of PD-L1 expression in a murine cell line by TPFS compounds. (A) The B16F10 mouse melanoma cell line was pre-stimulated with mouse IFN- $\gamma$ and cultured in the absence or presence of the indicated concentrations of TPFS-202. After $48 \mathrm{~h}$, cells were harvested and PD-L1 was analyzed using flow cytometry. Histograms (upper) and bar graphs of mPD-L1 median fluorescence intensities (lower) represent data acquired from three independent experiments. (B) B16F10 or RAW264.7 cells were stimulated with IFN- $\gamma$ and cultured in the absence or presence of various concentration of TPFS compounds for $24 \mathrm{~h}$, after which RNA was extracted and used for reverse transcription-quantitative PCR analysis. All samples were normalized to the expression of GAPDH. Data are presented as the fold change of mPD-L1 expression in comound-treated groups relative to the vehicle control. All graphs represent results from three independent experiments. " $\mathrm{P}<0.05$ vs. untreated controls. PD-L1, programmed death-ligand 1; IFN, interferon.

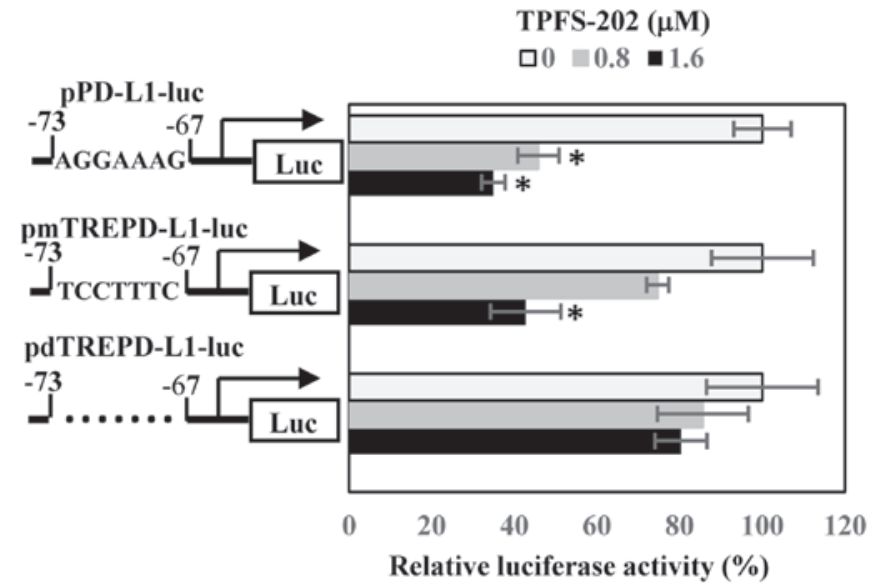

Figure 5. Involvement of putative TRE in TPFS-mediated PD-L1 inhibition. A549 cells stably transfected with reporter vectors under the control of PD-L1 promoter (pPD-L1-luc) or its mutants (pmTREPD-L1-luc and pdTREPD-L1-luc), in which the putative TRE was mutated or deleted as delineated at the left, were cultured in the absence or presence of the indicated concentrations of TPFS-202 for $48 \mathrm{~h}$. Cells were subsequently harvested and cell lysates were subjected to the luciferase assay and protein determination. The luciferase values were normalized with respect to protein content. Normalized luciferase activity from vehicle-treated cells was set as $100 \%$ and the values in the compound-treated groups are expressed as the relative percentage. The results presented are from three independent triplicate transfections. ${ }^{*} \mathrm{P}<0.05$ vs. untreated controls. TRE, TEAD response element; PD-L1, programmed death-ligand 1; pm, mutant; pd, deletion.

a novel member in the Hippo pathway-regulated gene network in human breast and lung cancer cell lines, and transcriptional regulation of PD-L1 by TAZ and YAP was not conserved in mouse cell lines. In view of this publication, it was postulated that the distinct effect of TPFS compounds on PD-L1 expression in human and mouse cell lines may be associated with a different regulation mechanism of PD-L1 by TAZ in these two species. Reporter vectors, in which the TRE was substituted (pmTREPD-L1-luc) or deleted (pdTREPD-L1-luc) to create a mutant PD-L1 promoter, were constructed to investigate this hypothesis (Fig. 5), and A549 cells stably transfected with these constructs were established. Consistent with data shown in Fig. 1C, treatment with TPFS-202 dose-dependently inhibited luciferase expression in cells with wild-type pPD-L1-luc plasmid, and disruption of TRE in the two mutant constructs, especially in pdTREPD-L1-luc, markedly abrogated the inhibition mediated by TPFS-202. This suggests that inhibition of PD-L1 by TPFS-202 is mediated, at least partially, by Hippo-TAZ/YAP signaling, although another pathway may also be involved in the observed inhibition of PD-L1.

\section{Discussion}

Immune checkpoint blockade has emerged as an effective treatment option for a wide range of tumor types, however, the objective tumor response is still limited to a fraction of cases and tumor types. Furthermore, monoclonal antibody (mAb)-based checkpoint inhibitors are associated with unique immune-related adverse events and high costs $(36,37)$. Therefore, there is an urgent requirement to explore alternative modalities based on non-mAb-based therapeutics, including small molecules. In the present study, the feasibility and 
therapeutic potential of amide analogues of brefelamide as small molecule immune checkpoint inhibitors by suppressing PD-L1 expression on tumor cells was investigated. The data presented in the current study revealed inhibitory effects of TPFS-201 and TPFS-202 on promoter activity, endogenous mRNA and PD-L1 surface protein expression, and inhibition of PD-L1 by the TPFS compounds consequently restored T cell activity, as evidenced by diminished apoptosis and increased IL-2 production from Jurkat T cells co-cultured with TPFS compound-treated A549 cells.

The majority of orthologous transcription factors play conserved roles in both human and mouse, however, some immune-related transcription regulation appear to be species specific $(38,39)$. A part of this regulation divergence in humans and mice is attributable to the differential expression of the transcriptional regulators in both species. Indeed, the expression pattern of transcriptional regulator, even master regulator, was found to be only partially conserved between humans and mice. Another possible mechanism for this difference in regulation between species is based on cis-regulatory elements being enriched in one species but not in the other $(40,41)$. In this regard, a recent study by Janse van Rensburg et al (29) has revealed species-specific regulation in the TAZ transcription program, and a couple of TAZ-regulated genes, including PD-L1 in human cells, were weakly responsive to TAZ overexpression in mouse cells, possibly due to difference in the cis-regulatory element of the promoter. In view of this distinction, it is not surprising that TPFS-mediated PD-L1 inhibition was blunted in mice cell lines. Considering the distinct regulation of PD-L1 by TAZ in the two species, it is reasonable to assume that PD-L1 inhibition by TPFS compounds may involve Hippo-TAZ signaling. The reporter assay performed in the present study revealed that disruption of the putative TAZ/TEAD-binding motif markedly abrogated the inhibition of PD-L1 by TPFS-202 (Fig. 5), suggesting a role for the Hippo-TAZ pathway in TPFS-mediated PD-L1 inhibition.

Increasing evidence has demonstrated YAP/TAZ-driven tumorigenesis in multiple types of solid tumors and highlighted a link between YAP/TAZ activation and cancer cell stemness, proliferation, chemoresistance and metastasis $(42,43)$. Thus, YAP/TAZ are emerging as promising therapeutic targets in malignant diseases. Further investigation of the effect of the brefelamide amide analogues on other downstream targets of Hippo signaling, besides PD-L1, may be warranted to determine if the compounds TPFS-201 and TPFS-202 are bona fide negative regulators of Hippo signaling. If confirmed, the anti-YAP/TAZ activities of these compounds have potential clinical relevance, and it may be worthy for further development of brefelamide amide analogues as promising therapeutic candidates for the malignancies associated with ablated YAP/TAZ activation.

Compared with those observed in pdTREPD-L1-luc, the inhibition effect of TPFS-202 was less attenuated by the substitution of TEAD responsive element in pmTREPD-L1-luc. While it is well known that transcription factors (TFs) bind to cis-regulatory elements with specific sequence preference, DNA structure is emerging as another important determinant of TF-DNA binding specificity. The mechanism in which TEAD/TAZ utilizes to recognize their cognate DNA in the PD-L1 promoter has not been fully elucidated, however, it is possible that the TEAD/TAZ-DNA interaction may be affected, not only by the primary nucleotide sequence, but by the structural features of the TEAD-binding sites in the PD-L1 promoter. The DNA structure crucial for TEAD-binding may be disrupted to a lesser extent in pmTREPD-L1-luc constructs compared with that in pdTREPD-L1-luc, which may account for their difference in respond to treatment with TFPS compounds.

The regulation of $\mathrm{PD}-\mathrm{L} 1$ expression is complex and multiple transcription regulators are involved. In addition to IFN- $\gamma$, a well-characterized stimulus for PD-L1 expression, recent evidence has linked multiple cell-intrinsic oncogenic pathways to PD-L1 expression in cancer cells. Among them are epidermal growth factor receptor (EGFR), mitogen-activated protein kinase kinase (MEK)-ERK and mitogen-activated protein kinase $\mathrm{p} 38$, the transcription factor MYC, and the kinase AKT (44-46). Thus, in addition to Hippo-TAZ/YAP signaling, the impact of the compounds TPFS-201 and TPFS-202 on these pathways may also contribute to the PD-L1 inhibition observed in the present study. Consistent with this hypothesis, it was found that disruption of the putative TRE did not fully abolished the PD-L1 inhibition by TPFS-202, the retained inhibition may partially be attributable to the effect of TPFS-202 on the oncogenic pathways aforementioned. Indeed, brefelamide was reported to inhibit phosphorylation of EGFR and attenuated EGFR-mediated ERK signaling cascade (27).

In addition to a critical role in the tumor immune evasion mechanism, PD-L1 has been reported to be involved in tumor proliferation, stemness, metastasis, and chemoresistance in multiple tumor types via tumor-intrinsic PD-L1 signaling (47-49), therefore it is reasonable to assume that besides attenuation of PD-1/PD-L1-mediated immunosuppression, the compounds TPFS-201 and TPFS-202 may exert extra-immune anti-tumor effect by downregulation of PD-L1 expression on tumor cells. Future work will be necessary to investigate this hypothesis. If confirmed, the amide analogues of brefelamide may be a promising therapeutic candidate used not only as an invigorator of immune response but as a monotherapy or in combination with conventional treatment to provide additive or synergistic anticancer effects.

The data presented in the present study suggest the effect of TPFS compounds on PD-L1 expression is species-specific. The presence of such species-related differences suggests that the predictive value of a conventional syngeneic mouse model is limited in evaluating the efficacy and safety of a therapeutic approach transcriptionally targeting PD-L1. In this regard, humanized mouse models, which are derived by engrafting human hematopoietic stem and progenitor cells into immunodeficient mice, may provide an alternative approach for this purpose.

In conclusion, the data presented in the present study indicate that the TPFS compounds suppressed PD-L1 expression and partially restored PD-1/PD-L1-mediated immunosuppression. These findings suggest the potential utility of brefelamide amide analogues as small molecule immune checkpoint inhibitors, thereby providing therapeutic alternatives, which can be used as monotherapy or combination with mAb-based blockade.

\section{Acknowledgements}

Not applicable. 


\section{Funding}

No funding was received.

\section{Availability of data and materials}

The datasets used and/or analyzed in the present study are available from the corresponding author on reasonable request.

\section{Authors' contributions}

JZ, OY, TH, YO and HK conceived and designed the study. JZ, OY, SK and SM performed the experiments. JZ and OY wrote the manuscript. All authors read and approved the manuscript.

\section{Ethic approval and consent to participate}

Not applicable.

\section{Patient consent for publication}

Not applicable.

\section{Competing interests}

The authors declare that they have no competing interests.

\section{References}

1. Zajac AJ, Blattman JN, Murali-Krishna K, Sourdive DJ, Suresh M, Altman JD and Ahmed R: Viral immune evasion due to persistence of activated $\mathrm{T}$ cells without effector function. J Exp Med 188: 2205-2213, 1998.

2. Gallimore A, Glithero A, Godkin A, Tissot AC, Plückthun A, Elliott $\mathrm{T}$, Hengartner $\mathrm{H}$ and Zinkernagel R: Induction and exhaustion of lymphocytic choriomeningitis virus-specific cytotoxic $\mathrm{T}$ lymphocytes visualized using soluble tetrameric major histocompatibility complex class I-peptide complexes. J Exp Med 187: 1383-1393, 1998.

3. Fuller MJ, Khanolkar A, Tebo AE and Zajac AJ: Maintenance, loss, and resurgence of T cell responses during acute, protracted, and chronic viral infections. J Immunol 172: 4204-4214, 2004

4. Wherry EJ: T cell exhausion. Nat Immunol 12: 492-499, 2011

5. Doering TA, Crawford A, Angelosanto JM, Paley MA Ziegler CG and Wherry EJ: Network and analysis reveals centrally connected genes and pathways involved in CD8+ T cell exhaustion versus memory. Immunity 37: 1130-1144, 2012.

6. Schietinger A and Greenberg PD: Tolerance and exhaustion: Defining mechanisms of T cell dysfunction. Trends Immunol 35: 51-60, 2014.

7. Butte MJ, Keir ME, Phamduy TB, Sharpe AH and Freeman GJ: Programmed death-1ligand 1 interacts specifically with the B7-1 costimulatory molecule to inhibit $\mathrm{T}$ cell responses. Immunity 27 : $111-122,2007$.

8. Freeman GJ, Long AJ, Iwai Y, Bourque K, Chernova T, Nishimura H, Fitz LJ, Malenkovich N, Okazaki T, Byrne MC, et al: Engagement of the PD-1 immunoinhibitory receptor by a novel $\mathrm{B} 7$ family member leads to negative regulation of lymphocyte activation. J Exp Med 192: 1027-1034, 2000.

9. Couzin-Frankel J: Breakthrough of the year 2013. Cancer immunotherapy. Science 342: 1432-1433, 2013.

10. Butt AQ and Mills KH: Immunosuppressive networks and checkpoints controlling antitumor immunity and their blockade in the development of cancer immunotherapeutics and vaccines. Oncogene 33: 4623-4631, 2014.

11. Merelli $\mathrm{B}$, Massi $\mathrm{D}$, Cattaneo L and Mandalà M: Targeting the PD1/PD-L1 axis in melanoma: Biological rationale, clinical challenges and opportunities. Crit Rev Oncol Hematol 89: 140-165, 2014.

12. Garon EB, Rizvi NA, Hui R, Leighl N, Balmanoukian AS, Eder JP, Patnaik A, Aggarwal C, Gubens M, Horn L, et al: Pembrolizumab for the treatment of non-small-cell lung cancer. N Engl J Med 372: 2018-2028, 2015.
13. Rosenberg JE, Hoffman-Censits J, Powles T, van der Heijden MS, Balar AV, Necchi A, Dawson N, O'Donnell PH, Balmanoukian A, Loriot Y, et al: Atezolizumab in patients with locally advanced and metastatic urothelial carcinoma who have progressed following treatment with platinum-based chemotherapy: A single-arm, multicentre, phase 2 trial. Lancet 387: 1909-1920, 2016.

14. Ansell SM, Lesokhin AM, Borrello I, Halwani A, Scott EC, Gutierrez M,Schuster SJ,Millenson MM, Cattry D, Freeman GJ, et al: PD-1 blockade with nivolumab in relapsed or refractory Hodgkin's lymphoma. N Engl J Med 372: 311-319, 2015.

15. Motzer RJ, Escudier B, McDermott DF, George S, Hammers HJ, Srinivas S, Tykodi SS, Sosman JA, Procopio G, Plimack ER, et al: Nivolumab versus everolimus in advanced renal-cell carcinoma. N Engl J Med 373: 1803-1813, 2015.

16. Caspi RR: Immunotherapy of autoimmunity and cancer: The penalty for success. Nat Rev Immunol 8: 970-976, 2008.

17. Amos SM, Duong CP, Westwood JA, Ritchie DS, Junghans RP, Darcy PK and Kershaw MH: Autoimmunity associated with immunotherapy of cancer. Blood 118: 499-509, 2011.

18. Lin DY, Tanaka Y, Iwasaki M, Gittis AG, Su HP, Mikami B, Okazaki T, Honjo T, Minato N and Garboczi DN: The PD-1/PD-L1 complex resembles the antigen-binding Fv domains of antibodies and T cell receptors. Pro Natl Acad Sci USA 105: 3011-3016, 2008.

19. Dong H, Zhu G, Tamada K and Chen L: B7-H1, a third member of the B7 family, co-stimulates T-cell proliferation and interleukin-10 secretion. Nat Med 5: 1365-1369, 1999.

20. Freeman GJ, Long AJ, Iwai Y, Bourque K, Chernova T, Nishimura H, Fitz LJ, Malenkovich N, Okazaki T, Byrne MC, et al: Engagement of the PD-1 immunoinhibitory receptor by a novel $\mathrm{B} 7$ family member leads to negative regulation of lymphocyte activation. J Exp Med 192: 1027-1034, 2000.

21. Dong H, Strome SE, Salomao DR, Tamura H, Hirano F, Flies DB, Roche PC, Lu J, Zhu G, Tamada K, et al: Tumor-associated B7-H1 promotes T-cell apoptosis: A potential mechanism of immune evasion. Nat Med 8: 793-800, 2002.

22. Ding H, Wu X and Gao W: PD-L1 is expressed by human renal tubular epithelial cells and suppresses T cell cytokine synthesis. Clin Immunol 115: 184-191, 2005.

23. Yang CY, Lin MW, Chang YL, Wu CT and Yang PC: Programmed cell death-ligand 1 expression in surgically resected stage I pulmonary adenocarcinoma and its correlation with driver mutations and clinical outcomes. Eur J Cancer 50: 1361-1369, 2014.

24. Gao Q, Wang XY, Qiu SJ, Yamato I, Sho M, Nakajima Y, Zhou J, Li BZ, Shi YH, Xiao YS, et al: Overexpression of PD-L1 significantly associates with tumor aggressiveness and postoperative recurrence in human hepatocellular carcinoma. Clin Cancer Res 15: 971-979, 2009.

25. Hino R, Kabashima K, Kato Y, Yagi H, Nakamura M, Honjo T, Okazaki T and Tokura Y: Tumor cell expression of programmed cell death-1 ligand 1 is a prognostic factor for malignant melanoma. Cancer 116: 1757-1766, 2010.

26. Kikuchi H, Saito Y, Sekiya J, Okano Y, Saito M, Nakahata N, Kubohara Y and Oshima Y: Isolation and synthesis of a new aromatic compound, brefelamide, from dictyostelium cellular slime molds and its inhibitory effect on the proliferation of astrocytoma cells. J Org Chem 70: 8854-8858, 2005.

27. Honma S, Saito M, Kikuchi H, Saito Y, OshimaY Nakahata N and Yoshida M: A reduction of epidermal growth factor receptor is involved in brefelamide induced inhibition of phosphorylation of ERK in human astrocytoma cells. Eur J Pharmacol 616: 38-42, 2009.

28. Zhang J, Yamada O, Kida S, Matsushita Y, Murase S, Hattori T, Kubohara Y,Kikuchi H and Oshima Y: Identification of brefelamide as a novel inhibitor of osteopontin that suppresses invasion of A549 lung cancer cells. Oncol Rep 36: 2357-2364, 2016.

29. Janse van Rensburg HJ, Azad T, Ling M, Hao Y, Snetsinger B, Khanal P, Minassian LM, Graham CH, Rauh MJ and Yang X: The hippo pathway component TAZ promotes immune evasion in human cancer through PD-L1. Cancer Res 78: 1457-1470, 2018.

30. Yamamoto R, Nishikori M, Tashima M, Sakai T, Ichinohe T, Takaori-Kondo A, Ohmori K and Uchiyama T: B7-H1 expression is regulated by MEK/ERK signaling pathway in anaplastic large cell lymphoma and Hodgkin lymphoma. Cancer Sci 100: 2093-2100, 2009.

31. Jiang X, Zhou J, Giobbie-Hurder A, Wargo J and Hodi FS: The activation of MAPK in melanoma cells resistant to BRAF inhibition promotes PD-L1 expression that is reversible by MEK and PI3K inhibition. Clin Cancer Res 19: 598-609, 2013.

32. Atefi M, Avramis E, Lassen A, Wong DJ, Robert L, Foulad D, Cerniglia M, Titz B, Chodon T, Graeber TG, et al: Effects of MAPK and PI3K pathways on PD-L1 expression in melanoma. Clin Cancer Res 20: 3446-3457, 2014. 
33. Mestas $\mathrm{J}$ and Hughes CC: Of mice and not men: Differences between mouse and human immunology. J Immunol 172: 2731-2738, 2004.

34. Davis MM: A prescription for human immunology. Immunity 29: 835-838, 2008

35. Hayday AC and Peakman M: The habitual, diverse and surmountable obstacles to human immunology research. Nat Immunol 9: $575-580,2008$.

36. Naidoo J, Page DB, Li BT, Connell LC, Schindler K, Lacouture ME, Postow MA and Wolchok JD: Toxicities of the anti-PD-1 and anti-PD-L1 immune checkpoint antibodies. Ann Oncol 26: 2375-2391, 2015.

37. Champiat S, Lambotte O, Barreau E, Belkhir R, Berdelou A, Carbonnel F, Cauquil C, Chanson P, Collins M, Durrbach A, et al: Management of immune checkpoint blockade dysimmune toxicities: A collaborative position paper. Ann Oncol 27: 559-574, 2016

38. Shay T, Jojic V, Zuk O, Rothamel K, Puyraimond-Zemmour D, Feng T, Wakamatsu E, Benoist C, Koller D and Regev A; ImmGen Consortium: Conservation and divergence in the transcriptional programs of the human and mouse immune systems. Proc Nat Acad Sci USA 110: 2946-2951, 2013.

39. Diehl AG and Boyle AP: Conserved and species-specific transcription factor co-binding patterns drive divergent gene regulation in human and mouse. Nucleic Acids Res 46: 1878-1894, 2018

40. Vierstra J, Rynes E, Sandstrom R, Zhang M, Canfield T, Hansen RS, Stehling-Sun S, Sabo PJ, Byron R, Humbert R, et al: Mouse regulatory DNA landscapes reveal global principles of cis-regulatory evolution. Science 346: 1007-1012, 2014.

41. Odom DT, Dowell RD, Jacobsen ES, Gordon W, Danford TW, MacIsaac KD, Rolfe PA, Conboy CM, Gifford DK and Fraenkel E: Tissue-specific transcriptional regulation has diverged significantly between human and mouse. Nat Genet 39: 730-732, 2007.
42. Harvey KF, Zhang X and Thomas DM: The Hippo pathway and human cancer. Nat Rev Cancer 13: 246-257, 2013.

43. Chen Q, Zhang N, Gray RS, Li H, Ewald AJ, Zahnow CA and Pan D: A temporal requirement for Hippo signaling in mammary gland differentiation, growth, and tumorigenesis. Genes Dev 28: 432-437, 2014.

44. Rech AJ and Vonderheide RH: Dynamic interplay of oncogenes and $\mathrm{T}$ cells induces PD-L1 in the tumor microenvironment. Cancer Discov 3: 1330-1332, 2013.

45. Parsa AT, Waldron JS, Panner A, Crane CA, Parney IF, Barry JJ, Cachola KE, Murray JC, Tihan T, Jensen MC, et al: Loss of tumor suppressor PTEN function increases B7-H1 expression and immunoresistance in glioma. Nat Med 13: 84-88, 2007.

46. Casey SC, Tong L, Li Y, Do R, Walz S, Fitzgerald KN, Gouw AM, Baylot V, Gütgemann I, Eilers M and Felsher DW: MYC regulates the antitumor immune response through CD47 and PD-L1. Science 352: 227-231, 2016.

47. Gao Q, Wang XY, Qiu SJ, Yamato I, Sho M, Nakajima Y, Zhou J, Li BZ, Shi YH, Xiao YS, et al: Overexpression of PD-L1 significantly associates with tumor aggressiveness and postoperative recurrence in human hepatocellular carcinoma. Clin Cancer Res 15: 971-979, 2009.

48. Zhang P, Ma Y, Lv C, Huang M, Li M, Dong B, Liu X, An G, Zhang $\mathrm{W}$, Zhang J, et al: Upregulation of programmed cell death ligand 1 promotes resistance response in non-small-cell lung cancer patients treated with neo-adjuvant chemotherapy. Cancer Sci 107: 1563-1571, 2016.

49. Gupta HB, Deng J, Clark CA, Drerup JM, Wu B, Sareddy G, Hurez V, Vadlamudi R, Li R and Curiel TJ: Programmed cell death ligand 1 (PD-L1) regulates tumor initiating cell (TIC) generation by controlling the stemness gene Oct4 through mTORC1. J Immunol 200 (1 Supplement): 167, 2018. 